\title{
Meibomian glands and contact lens wear
}

\author{
ANTONIO S. HENRIQUEZ1 AND DONALD R. KORB ${ }^{2}$ \\ From the ${ }^{1}$ Department of Ophthalmology, Ciudad Sanitaria Principes de España, \\ Barcelona, Spain, and the ${ }^{2}$ New England Optometric Association, Boston, Massachusetts, USA
}

SUMMARY A study of a syndrome characterised by deficient or inadequate Meibomian gland secretions, minimal or transient symptoms suggestive of ocular dryness, fluorescein staining of the cornea (often detected only after delayed observation or sequential instillation of stain), and contact lens intolerance is described. Clinical and cytological studies indicate that the syndrome is due to obstruction of the Meibomian gland orifices by desquamated epithelial cells that tend to aggregate in keratotic clusters, which results in alteration of the Meibomian glands' contribution to the precorneal tear film. Further complication may result from bacterial proliferation in the desquamated keratotic cells and the release of the bacteria and their toxic products into the precorneal tear film from these reservoirs in the excretory pathways of the Meibomian glands.

This study investigated the Meibomian glands of patients with the primary complaint of contact lens intolerance. Age and sex matched patients who showed optimal contact lens tolerance served as controls. We describe a syndrome characterised by deficient or inadequate Meibomian gland secretions, minimal or transient symptoms suggestive of ocular dryness, fluorescein staining of the cornea (often detected only after delayed observation or sequential instillation of $\left.\operatorname{stain}^{1}\right)$, and contact lens intolerance. The present communication reports the pathological findings from cytological and bacteriological examination of smears obtained before, during, and after sequential expression of the Meibomian glands of patients with the syndrome, and reports that a significant percentage of patients showing contact lens intolerance without obvious cause have this syndrome. A more detailed clinicopathological description of these studies is in press. ${ }^{2}$

\section{Materials and methods}

We investigated the Meibomian glands of 38 consecutive patients referred to us for evaluation of contact lens intolerance for which all conventional diagnostic techniques had failed to reveal a definitive cause. Twelve age and sex matched patients who showed optimal contact lens tolerance served as controls for bacteriological and cytological studies. Only the Meibomian glands of the lower

Correspondence to Dr A. S. Henriquez, Clinica Arruga, Pasaje Mendez Vigo 3, Barcelona-9, Spain. lid were studied. Samples of the Meibomian gland secretion were collected at the lid margin with the corner of a glass slide after gentle and forceful expression (Fig. 1). The sample was then smeared on to another glass slide, fixed for 20 minutes in (Harleco) Diff-Quik, and stained by the WrightGiemsa method for cytological study. Permanent slides were made by covering the sample with 1 drop of (Fisher Scientific) Permount histological mounting medium and a glass coverslip. These slides were viewed under a light microscope at 200,400 , and 1000 times magnification.

\section{Results and discussion}

The superficial oily layer of the tear film is primarily the result of Meibomian gland secretion. ${ }^{4}$ The Meibomian glands are located within the tarsal plate of the eyelids (Fig. 2), and belong to the category of holocrine glands (glands whose secretion is composed of entire cells that are released upon maturation). These cells originate in the most remote part of the glands. As they grow, their cytoplasm accumulates minute droplets of sebaceous material, which results in a characteristic foamy appearance (Fig. 3). At the same time the nucleus shrinks, and when the cell reaches full maturity the membrane ruptures and releases all its contents. In this way the sebaceous secretion is expelled into the excretory duct, where it mixes with the desquamated epithelial cells from the ductal lining.

Analysis of full-thickness biopsies of eyelids and tissue obtained from cadavers and exenteration 


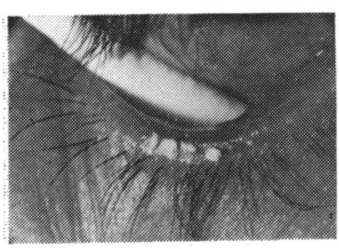

1

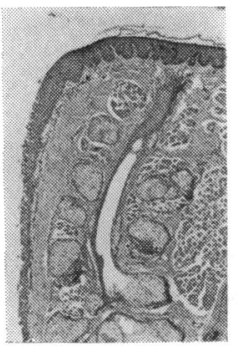

2

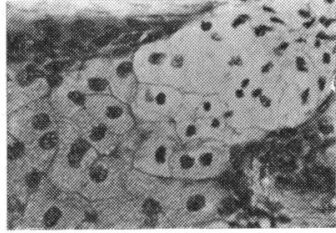

3

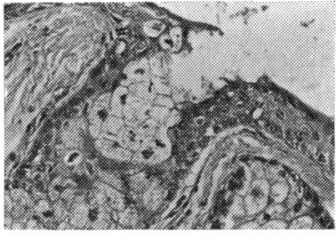

4 specimens reveals that the lining of the Meibomian glands' excretory ducts is made up of a multilayered squamous epithelium similar to that of the epidermis (Fig. 4). The product collected in the excretory duct is delivered to the tear film at the lid margin. If the orifice is either partially or totally obstructed, 2 problems may arise: stagnation and infection.

Stagnation of the sebaceous secretion, especially in the lower lid, may occur by external compression from an adjacent expanding mass (such as a granuloma or tumour) or by internal obstruction due to plugging of the excretory duct. Our concern will be directed to the latter. Certain skin diseases, such as seborrhoeic dermatitis and acne rosacea, are characterised by increased epithelial turnover. Large amounts of cells are produced, which detach from the epidermal surface in the form of small scales (Fig. 5). The mechanism is similar to that of dandruff production. The excretory duct of the Meibomian glands is lined by the same type of epithelium as the epidermal surface. Therefore it is reasonable to assume that obstruction of the duct is more likely under these circumstances of increased epithelial turnover because of the accumulation of desquamated epithelial cells-particularly near the lid margin (Fig. 6).

When stagnation occurs, the duct dilates (Fig. 7) because of the accumulation of sebaceous material and cellular debris from the Meibomian gland and the ductal lining. These changes are not always accompanied by pouting of the orifices or inflammatory signs (or both) along the lid margin, and the condition may easily be dismissed. In these circumstances, however, expression of the gland would release inspissated material containing numerous desquamated epithelial cells (Fig. 8), often aggregated in clusters or plugs (Fig. 9). Plugging of the excretory ducts of the Meibomian glands is usually insidious, often inapparent, and may lead to dysfunction and eventual atrophy of the gland. Clinically, stagnation of the Meibomian gland secretions has been said to be responsible for a form of superficial punctate keratitis similar to that seen in syndromes with a known unstable
Fig. 1 Clinical appearance of the lower lid margin in a patient with seborrhoeic blepharitis and obstruction of the Meibomian glands. Inspissated sebaceous secretion was obtained by expression. Note dryness on the lid margin surface.

Fig. 2 Histological section of the lower lid in an otherwise healthy young adult. The conjunctival surface is on the left. The excretory duct of a Meibomian gland is seen in the centre within the tarsal plate and surrounded by several glandular alveoli. The orifice of exit in the lid margin (top) is out of the plane of section. Note normal epidermal desquamation on the surface of the lid margin (top). (Haematoxylin-eosin; original magnification $\times 65$.)

Fig. 3 Photomicrograph of a Meibomian gland alveolus. The sebaceous material was dissolved at the time of histological processing leaving the cells with a typical foamy appearance. At maturity the nucleus shrinks and the cell membrane breaks, releasing all the cytoplasmic contents (upper right corner) into the excretory duct. (Haematoxylin-eosin; original magnification $\times 500$.)

Fig. 4 Photomicrograph illustrating the junction of a Meibomian gland alveolus with the excretory duct (upper right corner). Note the lining of the excretory duct by stratified squamous epithelium. (Haematoxylineosin; original magnification $\times 400$.)

tear film. ${ }^{5}$ Keratitis and blepharoconjunctivitis were also described ${ }^{6}$ in patients with Meibomian gland dysfunction. Abnormalities of Meibomian secretions have traditionally been considered an important cause of conjunctivitis. ${ }^{5}$ We are now presenting evidence that these patients with obstructed orifices or stagnated secretions, or both, show a significantly higher prevalence of contact lens intolerance.

The presence of dead, desquamated epithelial cells in the stagnated secretion of the Meibomian glands may provide an excellent culture medium for bacteria, as shown in this study (Fig. 10). The presence of bacteria in the smears was not always accompanied by clinical signs of inflammation on the lid margin or inflammatory cells in the collected Meibomian gland secretion. Bacteria were usually found to grow in small amounts only in dead, desquamated epithelial cells and not in neighbouring 


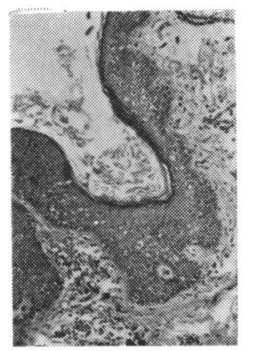

5

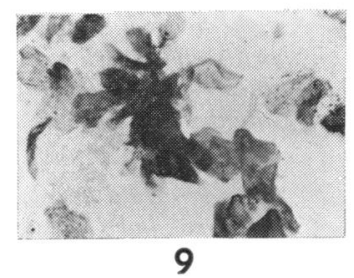

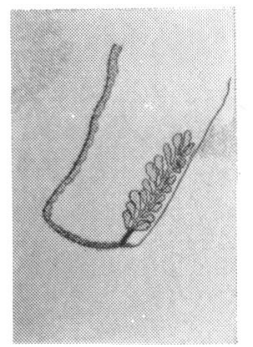

6

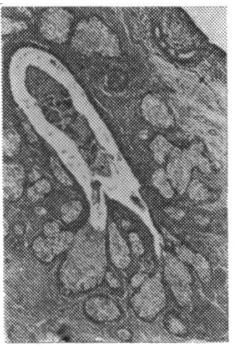

7

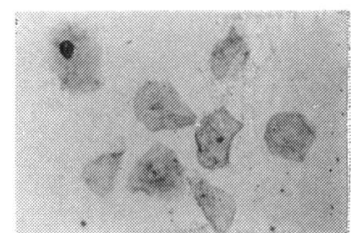

8

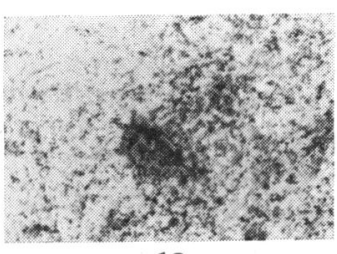

10

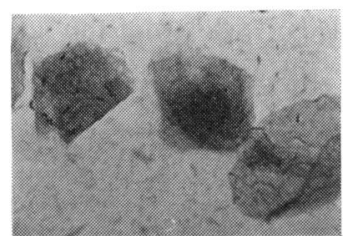

11 nucleated cells (Fig. 11). This finding may also explain why infections of the Meibomian glands are sometimes extremely resistant to either systemic or topical antibiotic treatment. Large amounts of bacteria were seen in smears from only 4 specimens from 4 patients who showed no inflammatory changes. However, the findings suggest the presence of a bacterial reservoir inside the Meibomian excretory pathways, which in turn may represent a continuous source of irritation to the anterior segment of the eye.

Several mechanisms of contact lens intolerance associated with Meibomian gland dysfunction are suggested. One appears to be due to the mechanical obstruction of the Meibomian glands by keratotic plugs, which probably results in an alteration of their oily secretion. The second mechanism may be related to the release of bacteria or their toxic products, or both, from the Meibomian glands into the precorneal tear film. The bacteriological studies indicated that the most frequent organisms identified were Staphylococcus epidermidis and Staphy. aureus. The role of these bacteria in causing inflammation in the anterior segment has been demonstrated. ${ }^{7} 8$

The possibility of Meibomian gland dysfunction is usually not investigated unless significant symptoms or gross signs are present. Symptoms are usually not present unless the integrity of the tear film is stressed, possibly by either a contact lens or by a dramatic change in the humidity or temperature of the environment. Thus, an apparently normal eye may unexpectedly show contact lens intolerance.

Treatment of Meibomian gland dysfunction should be directed toward relieving the obstruction of the ducts and orifices, thus allowing normal flow
Fig. 5 Histological section of the lid margin surface in a patient with increased epithelial turnover. Note the accumulation of keratotic cells on the epidermal surface. (Haematoxylin-eosin; original magnification $\times 250$.)

Fig. 6 The mechanism of obstruction of a Meibomian gland in certain skin diseases characterised by increased epidermal turnover is illustrated in this drawing: the desquamated epithelial cells from the skin surface and also from the inner ductal lining accumulate at the exit of the excretory duct with the formation of a keratotic plug and subsequent stagnation of the secretion inside the gland.

Fig. 7 Histological section of a stagnated Meibomian gland. Note the dilatation of the excretory duct, which is filled with sebaceous material and cell debris as compared with Fig. 2. Both pictures were obtained at the same magnification.

Fig. 8 Desquamated epithelial cells from the epidermal surface of the lid margin as recovered in a cytological smear with expression of Meibomian glands. (WrightGiemsa stain; original magnification $\times 500$.)

Fig. 9 Keratotic plug obtained after expression of Meibomian glands in a patient with seborrhoeic blepharitis. (Wright-Giemsa stain; original magnification $\times 500$.)

Fig. 10 Desquamated, dead epidermal cells contained within the stagnated Meibomian gland secretion may serve as an excellent culture medium for bacteria as illustrated in this smear. The entire field is filled with bacteria and cell debris. The cell in the centre is undergoing necrosis and destruction. (Wright-Giemsa stain; original magnification $\times 1000$.)

Fig. 11 Three epithelial cells scraped from the lid margin. A few bacteria are seen growing inside the 2 dead, non-nucleated squamous cells and not in the nucleated one (centre). (Wright-Giemsa stain; original magnification $\times 1000$.) 
of Meibomian gland secretion on to the precorneal tear film. Treatment is best accomplished by professional expressing of the Meibomian glands at appropriate intervals. Home therapy, consisting of hot compresses and scrubs of the lid margins, should be instituted on a daily basis, particularly in the initial stages.

\section{References}

1 Korb DR, Herman JP. Corneal staining subsequent sequential fluorescein instillations. $\mathrm{J}$ Am Optom Assoc $1978 ; 50$ : 361-7.
2 Korb DR, Henriquez AS. Meibomian gland dysfunction and contact lens intolerance. J Am Optom Assoc 1980; 51: 243-51.

3 Norn MS. Foam at outer palpebral canthus. Acta Ophthalmol (Kbh) 1963; 41: 531-7.

4 Ehlers N. The precorneal film. Acta Ophthalmol (Kbh) $1965 ; 43$ (suppl): 136-9.

5 McCulley JP, Sciallis GF. Meibomian keratoconjunctivitis. Am J Ophthalmol 1977; 84: 788-97.

6 Keith CG. Seborrheic blepharo-kerato-conjunctivitis. Trans Ophthalmol Soc UK 1967; 87: 85-103.

7 Thygeson P. Bacterial factors in chronic catarrhal conjunctivitis. Arch Ophthalmol 1937; 18: 373-80.

8 Allen JH. Staphylococcus conjunctivitis. Am J Ophthalmol $1937 ; 20$ : 1025-31. 